\title{
BMJ Open Parents and carers' experiences of seeking health information and support online for long-term physical childhood conditions: a systematic review and thematic synthesis of qualitative research
}

\author{
Bethan Mair Treadgold (D) , ${ }^{1}$ Emma Teasdale (D) , ${ }^{1}$ Ingrid Muller (D) , ${ }^{1}$ \\ Amanda Roberts, ${ }^{2}$ Neil Coulson, ${ }^{3}$ Miriam Santer ${ }^{1}$
}

To cite: Treadgold BM, Teasdale E, Muller I, et al. Parents and carers' experiences of seeking health information and support online for longterm physical childhood conditions: a systematic review and thematic synthesis of qualitative research. BMJ Open 2020;10:e042139. doi:10.1136/ bmjopen-2020-042139

- Prepublication history for this paper is available online To view these files, please visit the journal online (http://dx.doi org/10.1136/bmjopen-2020042139).

Received 30 June 2020 Revised 25 November 2020 Accepted 01 December 2020

D) Check for updates

(c) Author(s) (or their employer(s)) 2020. Re-use permitted under CC BY. Published by BMJ.

For numbered affiliations see end of article.

\section{Correspondence to} Bethan Mair Treadgold; b.m.treadgold@southampton. ac.uk

\section{ABSTRACT}

Objective To systematically review and synthesise qualitative research exploring parents/carers' experiences of seeking online information and support for long-term physical childhood conditions.

Design Systematic review and thematic synthesis of qualitative research.

Data sources Medline, CINAHL, Embase, PsycINFO and the International Bibliography of the Social Sciences were searched from inception to September 2019. We used thematic synthesis to analyse findings.

Eligibility criteria Primary research papers presenting qualitative data collection and analysis, focusing on parents/carers' experiences of seeking health information and support from online resources for long-term physical childhood health conditions. No language restrictions were placed.

Results 23 studies from seven countries met inclusion criteria and were included in the synthesis. Included studies presented data collected through interviews/ focus groups with 559 parents/carers; free-text surveys and essays with 26 parents/carers and 2407 messages from online support groups. Parents/carers developed a variety of strategies to obtain information and support online, based on personal preferences, appraisal of trustworthiness, perceived needs and previous experiences online. Many parents sought the benefits of online information and support, which included reassurance and validation from online communities, and feeling they had greater knowledge about their children's conditions. Some concerns and perceived risks were discussed, which often stemmed from prior unsatisfactory experiences of seeking information and support online, consultations with health professionals and seeing distressing stories online. Conclusion Most parents/carers were successful in obtaining information and support online. Many continued to share experiences with other parents/carers online. The need for information was particularly apparent early after diagnosis of the condition, whereas the need for peer support continued. The potential concerns and perceived risks with information and support online were especially apparent among parents/carers of children with lifelimiting long-term conditions. Findings may be useful for
Strengths and limitations of this study

- The first systematic review to synthesise the existing qualitative literature exploring parents/carers' experiences of seeking health information and support online for long-term physical childhood conditions.

- A comprehensive study synthesising the experiences of hundreds of parents/carers of children with a broad range of long-term physical conditions which provides new insights and develops the research field.

- A systematic and comprehensive search of the literature using multiple databases coupled with detailed synthesis of included studies.

- Excludes the online experiences of parents/carers of children with acute, mental health or developmental conditions and otherwise healthy children.

- Excluded quantitative studies which could have been used to support the qualitative findings.

health professionals to facilitate discussions regarding use of online resources, and researchers designing online health resources for parents/carers.

PROSPERO registration number CRD42018096009.

\section{BACKGROUND}

Caring for a child with a long-term condition can be challenging for parents and carers, requiring ongoing management with regular treatment use, and often complicated, timeconsuming and emotionally laden everyday routines. ${ }^{1}$ Seeking to maintain treatment and care regimes and dealing with social and financial constraints, while balancing the needs of the whole family, increase these challenges. $^{2}$ For the remainder of the paper, parents and carers are collectively referred to as 'parents'.

As a source of health information, the internet is convenient, instant and can help 
enhance knowledge and understanding of a condition. ${ }^{3}$ As a source of support, the internet also enables parents to exchange stories with other parents with similar experiences. This commonly occurs through asynchronous discussion platforms, ${ }^{4}$ although synchronous (real-time) discussions through online platforms are also increasingly popular. Reviews of qualitative research have explored how the internet influences peoples' health experiences. ${ }^{5}$ Experiences include obtaining health information to understand illness, redressing offline information and knowledge deficits, discussing experiences with health services, building connections with others, feeling supported and validated, releasing emotions and offline frustrations and learning self-management behaviours from others. Other reviews of qualitative research surrounding this area have explored the views of physicians regarding social media interventions to support child health, ${ }^{7}$ and experiences with health information online for carers of adults living with cancer. ${ }^{8}$ However, no systematic review has been conducted that synthesises the qualitative research on the online experiences of parents of children with long-term physical conditions.

\section{AIM}

This review aims to explore parents' experiences of seeking information and support online for long-term physical childhood conditions.

\section{METHOD}

This study follows the Centre for Reviews and Dissemination's guidance for undertaking reviews in healthcare, ${ }^{9}$ and the enhancing transparency in reporting the synthesis of qualitative research (ENTREQ) guidelines for reporting the synthesis of qualitative research. ${ }^{10}$

\section{Eligibility criteria}

We sought studies that explored parents' experiences of using the internet for information and support about long-term physical childhood condition(s) (table 1). To be eligible for inclusion, studies had to present qualitative data collection and analysis methods and qualitative data. Studies that used a mixed methodology were included if they comprised a qualitative component substantive enough to perform a qualitative analysis.

\section{Search strategy}

Five electronic databases were searched (Medline, CINAHL, Embase, PsycINFO and the International Bibliography of the Social Sciences) from the earliest date possible to September 2019. The Cochrane Library of Systematic Reviews and PROSPERO were also searched to check for any ongoing similar reviews. A comprehensive search strategy was developed with a medical librarian from the University of Southampton for each database (table 2). Search terms seeking to identify only qualitative research were initially included in search strategies, but we found that they did not improve the search

\begin{tabular}{|c|c|c|}
\hline & Inclusion criteria & Exclusion criteria \\
\hline Setting & $\begin{array}{l}\text { Online health information and support resources (eg, } \\
\text { websites, forums, social media pages, blogs) }\end{array}$ & $\begin{array}{l}\text { Primarily offline health information and support } \\
\text { resources (eg, family, friends, books, leaflets) }\end{array}$ \\
\hline \multirow[t]{2}{*}{ Design } & $\begin{array}{l}\text { Qualitative data collection methods (or mixed-method } \\
\text { studies involving qualitative data) (eg, interviews), } \\
\text { qualitative data analysis methods (eg, thematic } \\
\text { analysis) and presented qualitative data (eg, themes, } \\
\text { narratives and quotations) }\end{array}$ & Did not present the qualitative study criteria \\
\hline & $\begin{array}{l}\text { Primary research (eg, full research papers, feasibility } \\
\text { studies and pilot studies) }\end{array}$ & $\begin{array}{l}\text { Not primary research (eg, reviews, protocols and } \\
\text { commentaries) }\end{array}$ \\
\hline Population & $\begin{array}{l}\text { Parents and carers of children living with long-term } \\
\text { physical health conditions }\end{array}$ & $\begin{array}{l}\text { Not parents or carers (eg, family members without } \\
\text { caring responsibility for the child, health professionals } \\
\text { and teachers) }\end{array}$ \\
\hline \multirow{2}{*}{ Condition } & & $\begin{array}{l}\text { Developmental disorders of learning, language and } \\
\text { cognition (eg, attention deficit hyperactivity disorder) }\end{array}$ \\
\hline & & $\begin{array}{l}\text { Healthy populations or health-promoting related } \\
\text { studies (eg, diet, physical activity, obesity) }\end{array}$ \\
\hline Outcome & - Parents and carers' experiences & $\begin{array}{l}\text { Not parents or carers' experiences (eg, patterns of } \\
\text { internet usage derived from quantitative studies) }\end{array}$ \\
\hline
\end{tabular}




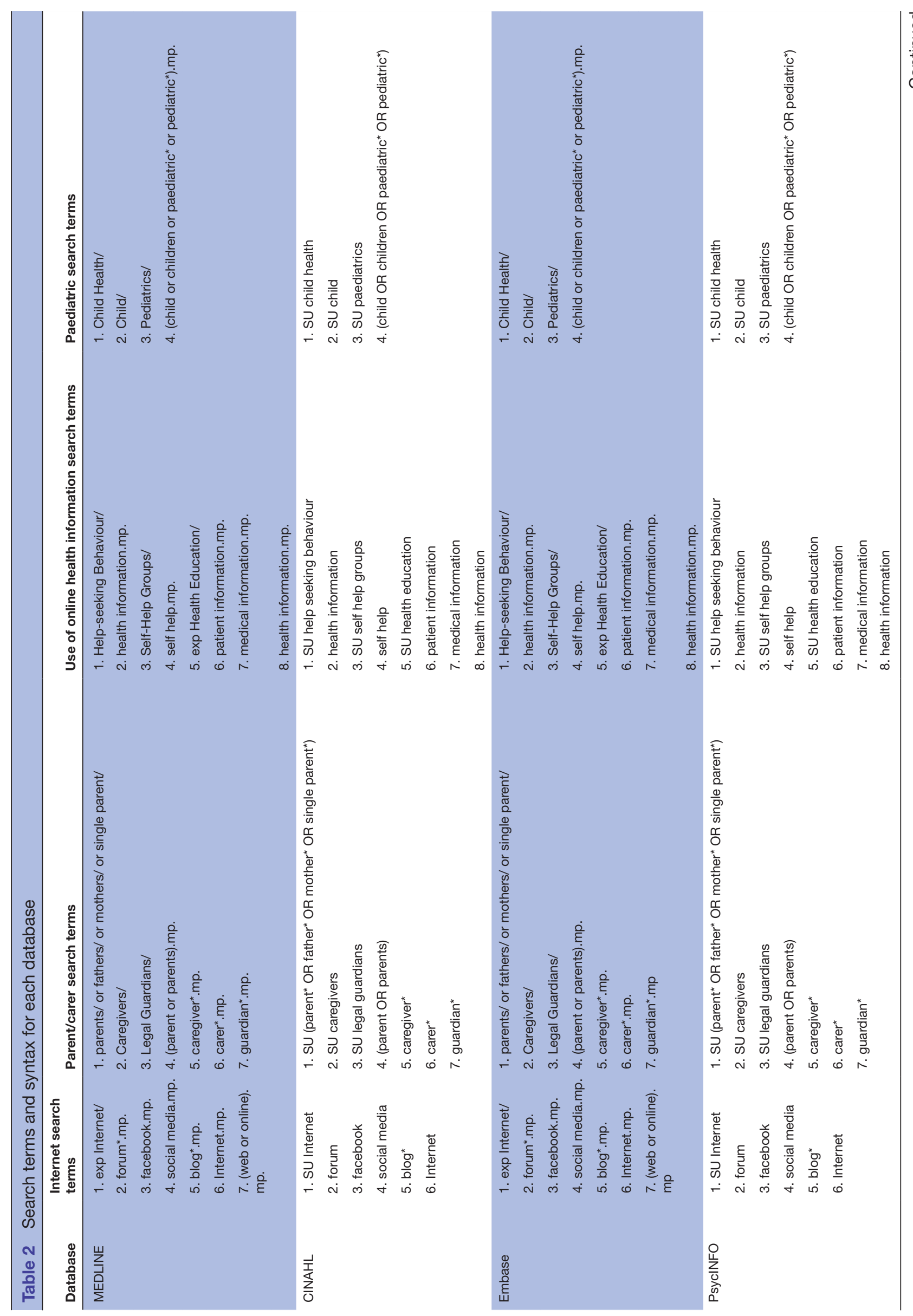




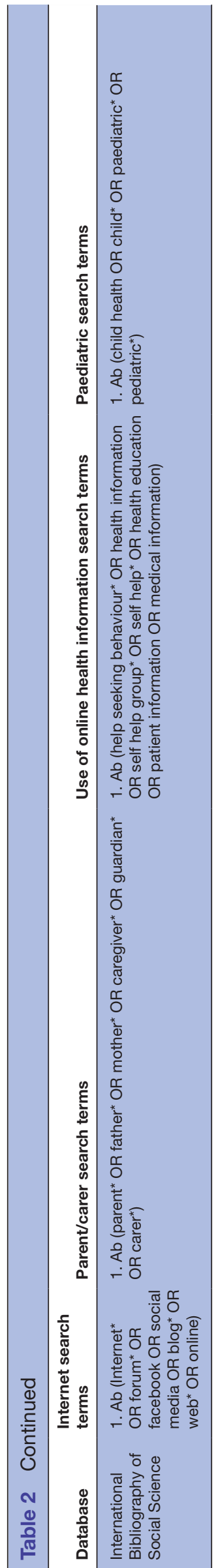

results so were subsequently removed. Others have similarly found subject-specific search strategies rather than methodology-based strategies to be more effective in identifying all relevant studies reporting qualitative findings. ${ }^{11}$

\section{Study screening methods}

One author (BMT) screened the titles and abstracts of all identified papers against the inclusion criteria. Duplicates of papers and those that failed to meet the inclusion criteria were eliminated during this stage. A second author (ET) screened $20 \%$ of the titles and abstracts to check for consistency. Full papers for the remaining identified studies were retrieved and assessed for eligibility (see figure 1).

\section{Quality appraisal and data extraction}

One author (BMT) extracted information from the included studies and tabulated it. Assessors (BMT, IM, MS, ET) independently assessed the quality of the included papers, using the Critical Appraisal Skills Programme quality assessment tool for qualitative research. ${ }^{12}$ Any discrepancies were discussed with the research team.

\section{Thematic synthesis}

We followed thematic synthesis for the analysis of the included studies, ${ }^{13}$ as this method has been successfully used for synthesising qualitative research in systematic reviews in health. ${ }^{14-19}$ Thematic synthesis consists of three stages. In stages 1 and 2, we explored and described the data. One author (BMT) read and re-read included papers, then inductively coded each line of the studies' findings according to their content and meaning to develop an initial coding frame. Participants' original data excerpts, as well as the authors' descriptions of findings, were coded in the latest version of NVivo. The coding frame was then discussed and refined within the research team (BMT, ET, IM, AR, NC, MS). In stage 3, we aimed to 'go beyond' individual study findings through developing descriptive themes initially and, subsequently, generating analytical themes.

\section{Patient and public involvement}

We involved an experienced public contributor with experience of eczema (AR) in the aim, design, analysis and write-up stages of the review. AR has a great deal of experience with online support groups, and has often highlighted that the online environment can be very supportive while also potentially bewildering. The contributor's experiences and preferences therefore helped shape the aim and design of the review: to explore parents' experiences of seeking information and support online for long-term physical childhood conditions.

\section{FINDINGS}

\section{Study selection}

The database searches resulted in 2981 records after excluding duplicates. A total of $23^{20-42}$ studies met 

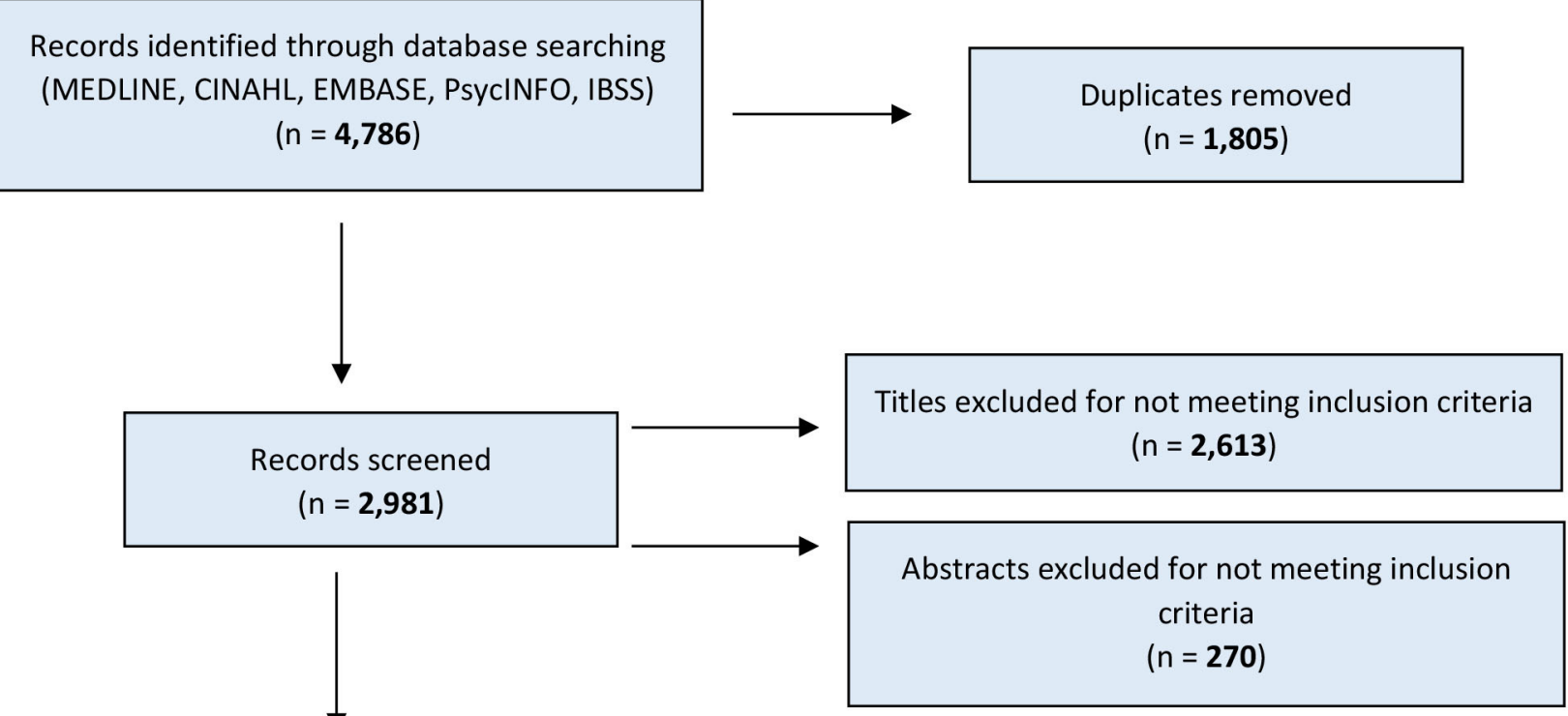

Full-text articles assessed for eligibility $(n=98)$

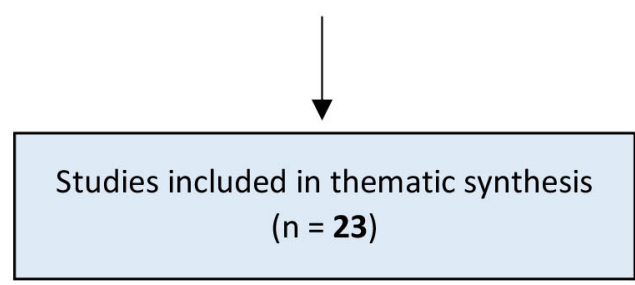

Full-text articles excluded $(n=75)$

- Not qualitative (38)

- Not primary research (11)

- Not focused on parents' experiences (8)

- Not focused on online health information (9)

- Not focused on a long-term physical health condition (5)

- Unable to access (4)

Figure 1 Preferred Reporting Items for Systematic Reviews and Meta-Analyses diagram of systematic search and study.

all inclusion criteria and were included for thematic synthesis (figure 1).

\section{Study characteristics}

All studies were published in English between December 2004 and July 2019, with the majority of studies $(n=14)$ being published since April 2016. A total of 11 studies were conducted in the Americas $\left(\mathrm{USA},{ }^{6} \mathrm{Canada},{ }^{4} \mathrm{Brazil}^{1}\right.$ ), 10 studies were conducted in Europe (UK, ${ }^{5}$ Sweden, ${ }^{2}$ The Netherlands ${ }^{2}$ and Norway ${ }^{1}$ ) and 2 studies were conducted in Australia. The 23 studies presented the experiences of 559 parents captured through interviews and focus groups, 26 parents through free-text surveys and essays and analyses of 2407 messages that had been posted by parents in online support groups (eg, online forums, social media sites and blogs), though it should be noted that not all studies provided sample size information (table 3).

\section{Quality of studies}

The quality assessment of the included studies revealed that all studies met most quality indicators. All studies had clear statements of the aims of the research, qualitative methodology was appropriate in all studies and was collected in a way that addressed the aims. We did not exclude any studies based on quality of reporting.

\section{Synthesis of results}

The papers included in this review broadly focused on how parents of children with long-term physical conditions use online resources for health information and support, and what they gain (or not) from using online resources. By synthesising the papers' findings, we interpreted three main analytical themes: (1) developing personal strategies for obtaining and appraising the online world; (2) feeling supported and informed by the online world and (3) perceived risk and concerns about the online world.

Figure 2 illustrates the analytical themes and descriptive themes, and table 4 lists all analytical and descriptive themes, along with references to the relevant papers.

How do parents develop personal strategies for obtaining and appraising information and support in the online world?

Parents were found to have personal preferences for certain types of websites and their content. ${ }^{21} 23$ 26-2831-42 Parents reported often favouring certain websites over others, which included official health information 

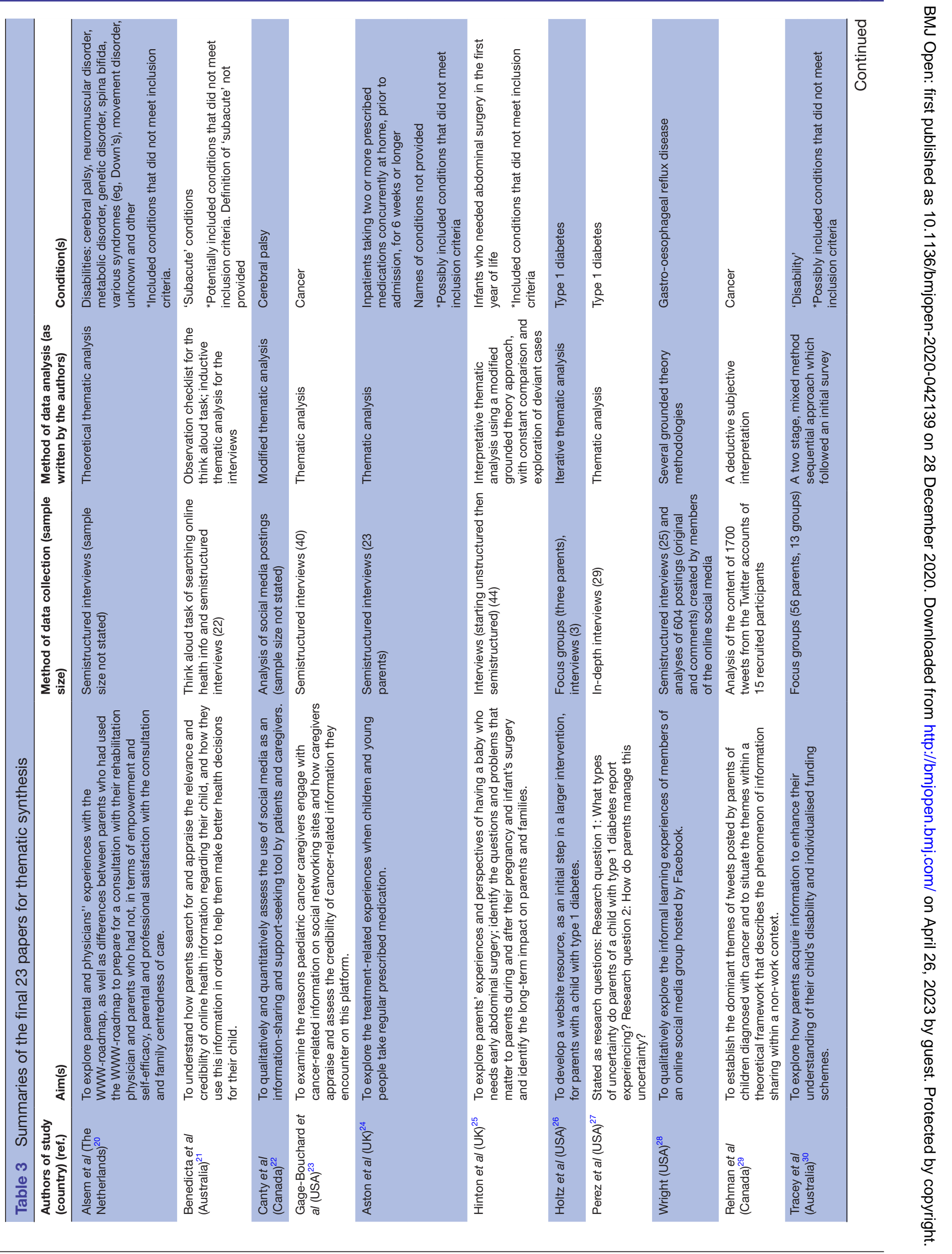


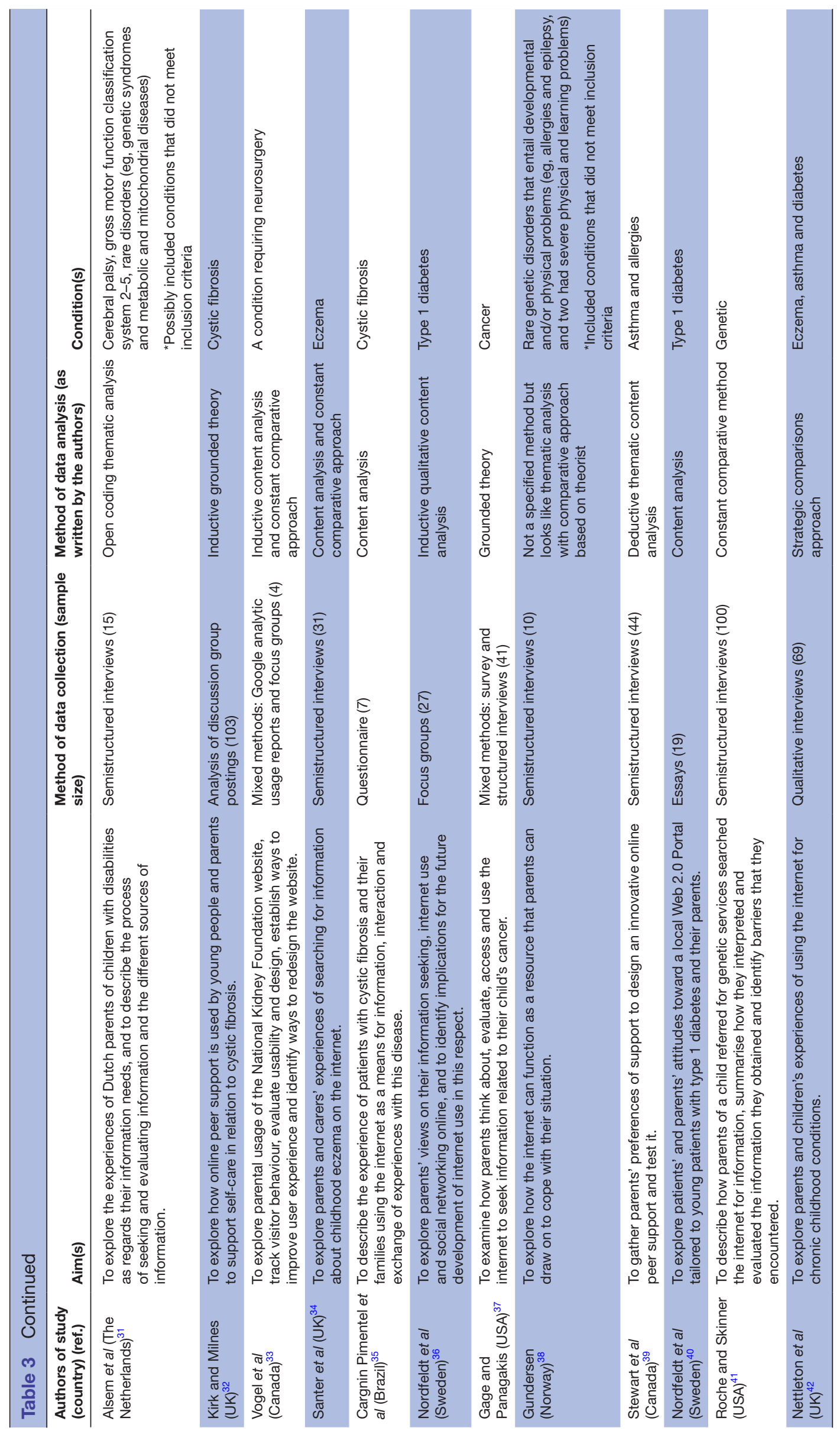




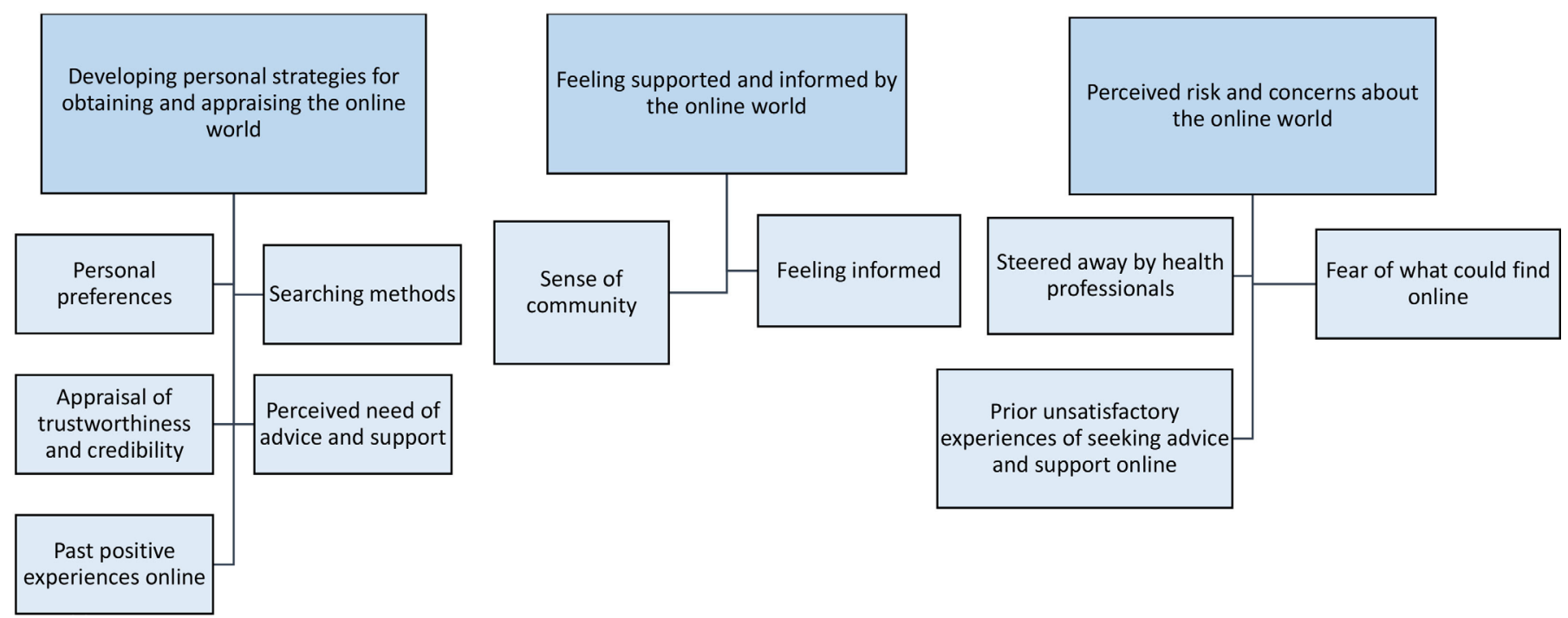

Figure 2 Illustration of the developed analytical themes and descriptive themes.

sites, charity websites, research sites, online discussion forums and social media sites. From within the different types of websites, parents also expressed informational preferences including medically moderated content, personalised advice and content dedicated to the condition without advertisements and other irrelevances. Parents also expressed functional preferences for online resources which included closed/private communities, live chat rooms and notifications for new content. 'For me, this is a

Table 4 Overview of analytical and descriptive themes across the presenting papers

Analytical theme Descriptive theme Paper references (total number)

Developing personal strategies for obtaining and appraising the online world

\begin{tabular}{|c|c|c|}
\hline & Personal preferences & $22,24,27,28,29,32,33,34,35,36,37,38,39,40,41,42,43(17)$ \\
\hline & Searching methods & $22,24,29,31,32,34,35,37,38,39,42,43(12)$ \\
\hline & $\begin{array}{l}\text { Appraisal of } \\
\text { trustworthiness and } \\
\text { credibility }\end{array}$ & $22,24,27,28,29,31,32,34,35,36,37,38,43(13)$ \\
\hline & $\begin{array}{l}\text { Perceived need of } \\
\text { information and } \\
\text { support }\end{array}$ & $21,22,23,26,27,28,29,33,34,35,36,37,38,39,40,41,42,43(18)$ \\
\hline & $\begin{array}{l}\text { Past positive } \\
\text { experiences online }\end{array}$ & $21,22,24,27,28,29,31,35,36,37,38,39,40,41,42,43(16)$ \\
\hline \multicolumn{3}{|l|}{$\begin{array}{l}\text { Feeling supported and } \\
\text { informed by the online world }\end{array}$} \\
\hline & Sense of community & $23,24,25,26,27,28,29,30,33,35,36,37,38,39,40,43(16)$ \\
\hline & $\begin{array}{l}\text { Prior unsatisfactory } \\
\text { experiences of } \\
\text { seeking information } \\
\text { and support online }\end{array}$ & $21,22,25,28,29,31,32,34,35,36,27,38,39,41,42,43(16)$ \\
\hline & $\begin{array}{l}\text { Steered away by } \\
\text { health professionals }\end{array}$ & $22,26,29,32,34,38(6)$ \\
\hline & $\begin{array}{l}\text { Fear of what could } \\
\text { find out online }\end{array}$ & $22,25,28,29,34,38,39,42(8)$ \\
\hline
\end{tabular}


funnel. This group funnels you. People can get quick answers, but if you want more in-depth information, we always do the link to a website because it has way more in-depth information than what you can get in the group online. ${ }^{28}$ (Gastro-oesophageal reflux disease, interview and online forum study)

Parents also described a variety of searching methods which enabled them to obtain online information and support. ${ }^{21} 23283031333436-384142$ Some visited the internet with a specific question in mind, others reported typing in keywords that they had heard during their medical consultations and others posted questions in a variety of online resources and combined the information with advice received offline. 'The same question on WhatsApp, Facebook and at the school. ${ }^{31}$ (Various conditions including cystic fibrosis, metabolic conditions and other disabilities, interview study)

Parents also reported appraising the trustworthiness and credibility of online resources, which influenced whether they decided to use the online resource. ${ }^{21}$ 23 26-28 30 31 33-37 42 Some had reported more specific tactics such as matching up information from a variety of websites, checking the affiliation/source of the website or the background of individual posters, the possible sales and marketing intentions behind the websites and comparing the advice with existing knowledge, whereas others implied that the reliability of online resources had been at least somewhat on their mind. '[The website] that came up first, they've probably got advertisements in there... everything is paid, so I am a bit sus on that. ${ }^{, 21}$ (Subacute conditions, think aloud and interview study)

Parents' perceived needs for information and support also appeared to influence whether and how they searched for information and support online. ${ }^{20-22}$ 25-28 32-42 This involved the present stage and severity of their child's condition, such as a recent diagnosis or a change in symptoms, and an unsatisfying recent medical consultation. 'Five minutes after we got the diagnosis... we got on the computer. I didn't know what else to do. ${ }^{41}$ (Genetic conditions, interview study)

Finally, parents also expressed past positive experiences with the online world, and how these often encouraged continuous use. ${ }^{2021} 23$ 26-28 30 34-42 Experiences included the internet's convenient and accessible nature for obtaining advice any time of the day, through a variety of mediums, for exploring sensitive topics and within the protection of anonymity and general successes of obtaining desired information. 'You know when you post even though it's like 5:00 in the morning, you've got other people that are also awake and even though it is 5:00 in the morning, they respond as well within a timely manner. ${ }^{, 28}$ (Gastrooesophageal reflux disease, interview and online forum study)

\section{How and why do parents feel supported and informed by the online world?}

The sense of community from using social media sites, discussion forums and blogs for peer support was also emphasised. ${ }^{22-29} 32$ 34-39 42 Connecting with other parents with similar experiences online was described as comforting, and often reduced their loneliness and sorrow. The importance of building trust and relationships in online communities was also reported, which enabled parents to gain more advice from one another, to share common language, to be treated as equals and to constantly be there to support one another. Parents also demonstrated that exchanging experiences online helped them to feel more reassured and validated about their situations. Also, parents expressed how online information and support that derives from others' experiences is invaluable, which often lacks in health professionals' advice.

...I'm so happy that I found this page. For me to share \& read about that there are other parents in my shoes. It breaks my heart watching my little girl try so hard to walk \& play with other kids... Her condition has really taken a toll on me. Physically, mentally, \& emotionally. I cry a lot. I'm just relieved there's parents on here that I can open up to. ${ }^{22}$ (Cerebral palsy, online forum study)

Parents across nearly all papers emphasised feeling informed through accessing and using information and support obtained online. ${ }^{20-26} 2829313235-42$ The sense of feeling better informed was reported to derive from parents' development of condition-related knowledge including information about diagnosis, prognosis, triggers and treatments. Parents often reported that their increase in medical knowledge helped them to feel in more control of managing their children's condition and to cope with stress and during uncertain times such as changes in symptoms and treatments. Accessing information online was also reported to facilitate parents in subsequent medical consultations, through helping them to feel more equipped to understand what their health professionals were telling them, to structure questions and to recommend plans for their children's treatments. Parents also reported feeling good about sharing their own experiences online about managing their children's conditions, such as with treatments, consultations and coping methods. 'I think it allows you to then make a decision on how serious something might be or whether you need to take action straight away or if it's something that you can potentially wait up and see what happens. ${ }^{21}$ (Subacute conditions, think aloud and interview study)

\section{How and why do parents develop concerns and perceive potential risks from seeking information and support online?}

While less common than positive accounts of feeling supported and informed, some parents reported the negative side to information and support online. Some parents reported that prior unsatisfactory experiences of seeking information and support online had discouraged them from using online resources. ${ }^{20} 2124$ 26-28 3031 33-35 3738 40-42 Previous experiences included not being able to find their desired information and support, being overwhelmed by 
the volume of content online, feeling that the internet does not replace real people and a lack of digital literacy skills and experience. 'I choose not to (go online) because there are so many diverse opinions out there. There's so much garbage out there. ${ }^{37}$ (Cancer, survey and interview study)

Parents also reported experiences of being steered away by health professionals from using the internet for information and support. ${ }^{21} 2528313337$ This was predominantly in papers that focused more on life-limiting conditions (eg, needing surgery in first year of life, gastrooesophageal reflux disease, cerebral palsy, neurological, cancer).

(Physician B) was very adamant, 'Don't you dare touch that internet, do not look at it, do not-you listen to what I say, I'm the boss, and this is the way it's going to run... I just wanted the definition ... I just wanted to know what the words meant.... ${ }^{33}$ (Conditions requiring neurosurgery, focus group study)

Finally, some parents reported that the fear of what they could find out online prevented them from using online resourcesforinformation and support. ${ }^{2124272833373841}$ Some reported having read distressing stories in the past, and others simply did not want to risk uncovering distressing information that they could relate to. These papers also focused more on life-limiting conditions (cancer, type 1 diabetes, rare genetic conditions, neurological).

I was careful with going online because I think the internet can be a really scary place. We got into a couple of those forums that tend to be sort of doom and gloom and focus on all the negative things. It's all the people that are struggling the most that seem to post in some of those forums. ${ }^{27}$ (Type 1 diabetes, interview study)

\section{DISCUSSION}

\section{Summary of findings}

This systematic review and thematic synthesis revealed that parents developed a variety of strategies to obtain and appraise information and support online in the context of caring for long-term physical childhood conditions. These processes were based on their personal preferences, their search methods, the outcome of their appraisal of trustworthiness, the degree of their perceived need for information and support and previous positive experiences online. In most cases, parents reported going on to experience the benefits of accessing information and support online. This included the sense of community gained through connecting with others in a similar situation online, building relationships and trust and feeling reassured and validated, which was available from online support communities especially. Parents also reported feeling better informed about their children's conditions, which helped them feel more in control in subsequent medical consultations and felt good about helping similar others by sharing their own experiences. However, some parents also emphasised the concerns and perceived risks of obtaining and using information and support online. These often stemmed from prior unsuccessful online experiences, having been told to avoid the internet by health professionals, and due to the fear of the distressing stories that they might come across online.

\section{Findings in context of existing research}

The findings from this study are largely consistent with other reviews that have explored peoples' experiences with health information online,${ }^{56}$ although these reviews had not focused specifically on the online experiences of parents of children with long-term physical conditions. However, our review uncovered more about the initial processes through which parents developed search strategies to obtain and evaluate health information and support online and the motivations behind these, which lead to the subsequent positive and negative experiences identified. Our review also drew more attention to the negative experiences that parents experienced online compared with these reviews, ${ }^{56}$ which could be due to the additional challenges of caring for a child rather than self-caring.

Collating and synthesising the experiences of parents of children with a variety of long-term conditions provided additional insights into parents' experiences with the online world. Drawing on the different experiences discussed suggested that the potential harms of the online world were most apparent for parents of children with life-limiting conditions, or whose conditions were unstable (eg, cerebral palsy, cancer, type 1 diabetes, rare genetic conditions, gastro-oesophageal reflux disease). Concerns and perceived risks were not found in the papers focusing on eczema, asthma and other allergies and other disabilities. This suggests that parents of children with life-limiting or unstable conditions use information and support online with more caution.

This review was also able to further identify that the types of information and support sought by parents differed according to the stage and severity of their children's conditions. The need for condition-related information was again more apparent across the papers that focused on the life-limiting or unstable conditions, whereas the need for peer support was apparent across all stages and severities (life-limiting, uncertain to stable and well managed). This suggests that parents' need for condition-specific information comes first and is not needed in long term, whereas the need for peer support is continuous.

\section{Strengths and limitations}

This is the first systematic review to synthesise the existing qualitative literature exploring parents' experiences of seeking health information and support online for long-term physical childhood conditions, providing new insights and developing the research field in this area. 
A limitation to this study was that some of the included studies for synthesis also explored parents' online experiences for conditions that are not long term and physical (eg, acute conditions, disabilities of learning, language and cognition, mental health conditions and health promotion in childhood), within the same paper. Some of the findings in these papers were not separated according to different conditions, therefore this review fails to identify possible differences between the online experiences of parents of children with long-term physical conditions and other types of conditions. However, this was never an aim of the study. Some included papers had limitations in reporting of methodological detail, for instance, sample size information. Additionally, included studies typically presented limited demographic data so it was not possible to explore how such factors may affect parents' experiences in the online world. We also excluded quantitative studies which could have been used to support the qualitative findings, and did not search the grey literature which could have excluded some relevant studies. Finally, we analysed only the results sections of each paper, meaning that broader contextual factors that may have arisen in the discussion sections were not included although the discussion sections were read and checked in case.

\section{Implications and future research}

Improved understanding of parents' experiences of seeking health information and support online for longterm physical childhood conditions may help health professionals treating these families. The findings from this study could be used to inform health professionals about parents' experiences online: such as what they want from information and support online, why they choose online resources, what they are influenced by and the types of information and support that they access online.

Parents' preferences and perceived benefits of online resources could be useful for future studies designing online resources for parents of children with long-term conditions. Specific examples that this study identified were online resources that contain medically moderated content, reminders to use online resources, frequently active members to reply to posts, easy to use and information tailored to the child's condition and its severity. A future systematic review could collate which online resources for certain conditions are evidence based and meet the needs of parents, so that health professionals can signpost patients to such online resources.

\section{CONCLUSION}

This systematic review and synthesis of qualitative literature comprehensively explored parents' experiences with health information online for long-term physical childhood conditions. This highlights the support that parents receive from online resources, but also their perceptions of potential risks and the processes by which they attempt to establish trustworthiness of sources. These findings could help health professionals to understand parents' perspectives about information and support online, and potentially help them identify robust resources that also meet patients' needs.

Author affiliations

${ }^{1}$ Primary Care, Population Sciences and Medical Education, Faculty of Medicine, University of Southampton, Southampton, UK

${ }^{2}$ Centre of Evidence-Based Dermatology, Faculty of Medicine and Health Sciences, University of Nottingham, Nottingham, UK

${ }^{3}$ Division of Rehabilitation, Ageing and Wellbeing, Faculty of Medicine and Health Sciences, University of Nottingham, Nottingham, UK

Twitter Bethan Mair Treadgold @BethanTreadgold and Ingrid Muller @IngridMuller7 Acknowledgements We would like to thank Paula Sands for her expertise in developing the search strategy for this study.

Contributors BMT developed and refined the protocol, undertook the systematic review, carried out data extraction, quality assessment and thematic synthesis and drafted the manuscript. ET refined the protocol, carried out eligibility screening process, quality assessment and thematic synthesis and wrote the manuscript. MS and IM refined the protocol, carried out quality assessment and thematic synthesis and wrote the manuscript. AR and NC refined the protocol, contributed to interpretation of findings and wrote the manuscript. All authors read and approved the final manuscript.

Funding This study was part of a PhD studentship funded by the National Institute for Health Research (NIHR) School for Primary Care Research. The views expressed are those of the authors and not necessarily those of the NIHR or the Department of Health and Social Care.

Competing interests None declared.

Patient consent for publication Not required.

Provenance and peer review Not commissioned; externally peer reviewed.

\section{Data availability statement No data are available.}

Open access This is an open access article distributed in accordance with the Creative Commons Attribution 4.0 Unported (CC BY 4.0) license, which permits others to copy, redistribute, remix, transform and build upon this work for any purpose, provided the original work is properly cited, a link to the licence is given, and indication of whether changes were made. See: https://creativecommons.org/ licenses/by/4.0/.

\section{ORCID iDs}

Bethan Mair Treadgold http://orcid.org/0000-0002-0255-7422

Emma Teasdale http://orcid.org/0000-0001-9147-193X

Ingrid Muller http://orcid.org/0000-0001-9341-6133

\section{REFERENCES}

1 Eiser C. Chronic childhood disease: an introduction to psychological theory and research. Cambridge University Press, 1990: 27.

2 Smith J, Cheater F, Bekker H. Parents' experiences of living with a child with a long-term condition: a rapid structured review of the literature. Health Expect 2015;18:452-74.

3 Wainstein BK, Sterling-Levis K, Baker SA, et al. Use of the Internet by parents of paediatric patients. $J$ Paediatr Child Health 2006;42:528-32.

4 Plantin L, Daneback K. Parenthood, information and support on the internet. A literature review of research on parents and professionals online. BMC Fam Pract 2009;10:1-2.

5 Ziebland S, Wyke S. Health and illness in a connected world: how might sharing experiences on the internet affect people's health? Milbank Q 2012;90:219-49.

6 Allen C, Vassilev I, Kennedy A, et al. Long-term condition selfmanagement support in online communities: a meta-synthesis of qualitative papers. J Med Internet Res 2016;18:e61.

7 Hamm MP, Shulhan J, Williams G, et al. A systematic review of the use and effectiveness of social media in child health. BMC Pediatr 2014;14:138.

8 Kinnane NA, Milne DJ. The role of the Internet in supporting and informing carers of people with cancer: a literature review. Support Care Cancer 2010;18:1123-36. 
9 Dissemination CF. Systematic reviews: CRD's guidance for undertaking reviews in healthcare. York: University of York NHS Centre for Reviews \& Dissemination, 2009.

10 Tong A, Flemming K, Mclnnes E, et al. Enhancing transparency in reporting the synthesis of qualitative research: ENTREQ. BMC Med Res Methodol 2012;12:181.

11 Gorecki CA, Brown JM, Briggs M, et al. Evaluation of five search strategies in retrieving qualitative patient-reported electronic data on the impact of pressure ulcers on quality of life. $J$ Adv Nurs 2010;66:645-52.

12 Critical Skills Appraisal Programme. CASP qualitative checklist, 2018

13 Thomas J, Harden A. Methods for the thematic synthesis of qualitative research in systematic reviews. BMC Med Res Methodol 2008;8:45.

14 Santer M, Ring N, Yardley L, et al. Treatment non-adherence in pediatric long-term medical conditions: systematic review and synthesis of qualitative studies of caregivers' views. BMC Pediatr 2014;14:63.

15 Morton RL, Tong A, Howard K, et al. The views of patients and carers in treatment decision making for chronic kidney disease: systematic review and thematic synthesis of qualitative studies. $B M J$ 2010;340:c112

16 Joseph-Williams N, Elwyn G, Edwards A. Knowledge is not power for patients: a systematic review and thematic synthesis of patientreported barriers and facilitators to shared decision making. Patient Educ Couns 2014:94:291-309.

17 Hefferon K, Grealy M, Mutrie N. Post-traumatic growth and life threatening physical illness: a systematic review of the qualitative literature. Br J Health Psychol 2009;14:343-78.

18 Pocock M, Trivedi D, Wills W, et al. Parental perceptions regarding healthy behaviours for preventing overweight and obesity in young children: a systematic review of qualitative studies. Obes Rev 2010:11:338-53.

19 Bridges J, Flatley M, Meyer J. Older people's and relatives' experiences in acute care settings: systematic review and synthesis of qualitative studies. Int J Nurs Stud 2010;47:89-107.

20 Alsem MW, Verhoef M, Braakman J, et al. Parental empowerment in paediatric rehabilitation: exploring the role of a digital tool to help parents prepare for consultation with a physician. Child Care Health Dev 2019;45:623-36.

21 Benedicta B, Caldwell PH, Scott KM. How parents use, search for and appraise online health information on their child's medical condition: a pilot study. J Paediatr Child Health 2020;56:252-8.

22 Canty MJ, Breitbart S, Siegel L, et al. The role of social media in selective dorsal rhizotomy for children: information sharing and social support. Childs Nerv Syst 2019;35:2179-85.

23 Gage-Bouchard EA, LaValley S, Devonish JA. Deciphering the signal from the noise: caregivers' information appraisal and credibility assessment of cancer-related information exchanged on social networking sites. Cancer Control 2019;26:107327481984160.

24 Aston J, Wilson KA, Terry DRP. The treatment-related experiences of parents, children and young people with regular prescribed medication. Int J Clin Pharm 2019;41:113-21.

25 Hinton L, Locock L, Long A-M, et al. What can make things better for parents when babies need abdominal surgery in their first year of life? A qualitative interview study in the UK. BMJ Open 2018;8:e020921.
26 Holtz BE, Mitchell KM, Nuttall AK, et al. Using user-feedback to develop a website: MyT1DHope, for parents of children with T1D. Health Commun 2020;35:281-8.

27 Perez L, Romo LK, Bell T. Communicatively exploring uncertainty management of parents of children with type 1 diabetes. Health Commun 2019;34:949-57.

28 Wright JV. Parents caring, sharing, and learning together online: an exploratory look at informal learning via a health-related support group in Facebook. Arizona State University, 2018.

29 Rehman S, Lyons K, McEwen R, et al. Motives for sharing illness experiences on Twitter: conversations of parents with children diagnosed with cancer. Inform Commun Soc 2018;21:578-93.

30 Tracey D, Johnston C, Papps FA, et al. How do parents acquire information to support their child with a disability and navigate individualised funding schemes? J Res Spl Educat Need 2018;18:25-35.

31 Alsem MW, Ausems F, Verhoef M, et al. Information seeking by parents of children with physical disabilities: an exploratory qualitative study. Res Dev Disabil 2017;60:125-34.

32 Kirk S, Milnes L. An exploration of how young people and parents use online support in the context of living with cystic fibrosis. Health Expect 2016;19:309-21.

33 Vogel TK, Kleib M, Davidson SJ, et al. Parental evaluation of a nurse practitioner-developed pediatric neurosurgery website. JMIR Res Protoc 2016;5:e55.

34 Santer M, Muller I, Yardley L, et al. 'You don't know which bits to believe': qualitative study exploring carers' experiences of seeking information on the internet about childhood eczema. BMJ Open 2015:5:e006339.

35 Cargnin Pimentel ED, dos Santos Luz G, Marisa Pelloso S, et al. Using the internet to exchange information and experience on cystic fibrosis. Investigación y Educación en Enfermería 2013;31:457-63.

36 Nordfeldt S, Ängarne-Lindberg T, Nordwall M, et al. Parents of adolescents with type 1 diabetes--their views on information and communication needs and internet use. A qualitative study. PLOS One 2013;8:e62096.

37 Gage EA, Panagakis C. The devil you know: parents seeking information online for paediatric cancer. Sociol Health IIIn 2012;34:444-58

38 Gundersen T. 'One wants to know what a chromosome is': the Internet as a coping resource when adjusting to life parenting a child with a rare genetic disorder. Sociol Health IIIn 2011;33:81-95.

39 Stewart M, Letourneau N, Masuda JR, et al. Online solutions to support needs and preferences of parents of children with asthma and allergies. J Fam Nurs 2011;17:357-79.

40 Nordfeldt S, Hanberger L, Berterö C. Patient and parent views on a Web 2.0 Diabetes Portal--the management tool, the generator, and the gatekeeper: qualitative study. J Med Internet Res 2010;12:e17.

41 Roche MI, Skinner D. How parents search, interpret, and evaluate genetic information obtained from the Internet. $J$ Genet Couns 2009;18:119-29.

42 Nettleton S, Burrows R, O'Malley L, et al. Health E-types' information. Communicat Soc 2004;7:531-53.

43 Department of Health and Social Care. Policy paper: 2010 to 2015 government policy: long term health conditions, 2015from: Available: https://www.gov.uk/government/publications/2010-to2015-government-policy-long-term-health-conditions/2010-to-2015government-policy-long-term-health-conditions\#issue [Accessed 22 June 2020]. 